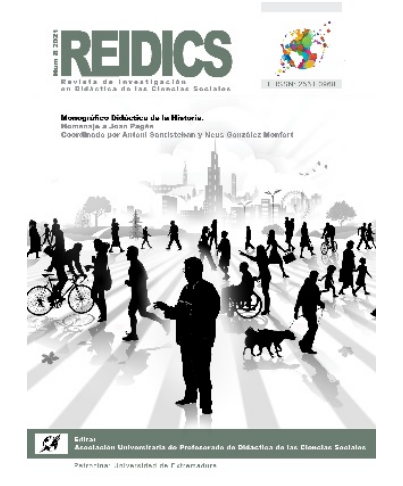

\title{
REIDICS
}

Revista de Investigación en

Núm. 8, 2021

Didáctica de las Ciencias

Sociales

Recibido 30 de junio de 2020

Aceptado 22 de febrero 2021

E-ISSN: 2531-0968

\section{El ensayo como instrumento para la formación docente en Educación para la Ciudadanía y Derechos Humanos}

\author{
Essay as a tool for teacher training in Education for Citizenship \\ and Human Rights
}

Aurora Arjones Fernández

Universidad de Málaga

Email:maarjones@uma.es

ORCID: https://orcid.org/0000-0003-4415-7125

Anabel Fernández Moreno

Universidad de Málaga

Email: anabel.femo@uma.es

ORCID: https://orcid.org/0000-0002-8410-9729

DOI: https://doi.org/10.17398/2531-0968.08.190

\section{Resumen}

Este artículo forma parte de un proyecto de innovación educativa en la formación del profesorado de Educación para la Ciudadanía y los Derechos Humanos, en el que participa el 54\% de alumnado de 3o de Grado de Educación Primaria de la UMA (2019/20). Aquí se sintetizan las primeras conclusiones de una investigación en curso sobre la redacción de ensayos como principal actividad de aprendizaje para desarrollar la capacidad de análisis en la enseñanza superior. Para ello, en primer lugar, repasamos la importancia del desarrollo del pensamiento crítico en los maestros en formación. Después, revisamos el estado de la cuestión respecto al uso del ensayo en el aula universitaria para descubrir que ninguno de los estudios consultados lo utilizó en esta disciplina. Por último, abordamos la pregunta de investigación: ¿es el ensayo un instrumento ágil para la formación de estructuras de pensamiento crítico en los futuros docentes? El análisis cualitativo mostró que, el ensayo como instrumento para la formación docente fue una herramienta innovadora y efectiva. Pudimos comprobar como la investigación autónoma y la escritura de ensayo: 1) mejoró las habilidades asociadas al pensamiento crítico, 2) ayudó a alcanzar competencias para la vida laboral, mediante la integración de prácticas reflexivas, 3) innovó en la apropiación del conocimiento, poniendo énfasis en el aprendizaje centrado en el alumnado, y 4) favoreció el desarrollo de estrategias de información, sensibilización, conciencia y empoderamiento. Sin embargo, también 
identificamos deficiencias en cuanto a las habilidades de escritura y argumentación que podrían mejorarse mediante la realización de talleres específicos.

Palabras clave: pensamiento crítico; prensa; educación para la ciudadanía; ensayo; derechos humanos.

\begin{abstract}
This paper is a part of an educational innovation project in the training of teachers of Citizenship Education and Human Rights, which participated 54\% of the 3rd undergraduate students in UMA's Primary Education (2019/20). We summarise the first findings of on-going research on essay-writing as the primary learning activity for developing higher education analytical skills. Firstly, we discuss the importance of promoting critical thinking (CT). Secondly, we review the state-of-the-art using essay-writing in the university classroom. None of the studies consulted did it in this discipline. Finally, we approach our research question: is essay-writing a suitable tool for critical thinking (CT) training for Education for Citizenship and Human Rights' primary education teachers? Qualitative analysis of the students' work showed that essay-writing as a tool for teacher training was an innovative tool. Furthermore, we were able to verify how independent research and essay-writing: 1) improved the skills associated with critical thinking, 2) helped to achieve competences for working life through the integration of reflective practices, 3) innovated in the appropriation of knowledge, emphasising student-centred learning, and 4) helped the development of strategies for information, awareness, consciousness and empowerment. However, it also identified shortcomings concerning the students' writing and argumentative skills that we could improve by implementing specific workshops.
\end{abstract}

Keywords: critical thinking; press; education for citizenship; essay; human rights

\title{
1. Introducción
}

La comunidad acepta y distingue cada profesión a partir del beneficio que esta ofrece al proyecto social. Ciertamente, el maestro tiende a reconocerse en "el bien que aporta a la sociedad" cuya función es "transmitir cultura y conocimientos y formar personas críticas y autónomas" (Cortina, 2013, p. 136). Por eso, mejorar la capacidad del docente para pensar de forma crítica y analizar las cuestiones que preocupan a la sociedad se considera fundamental en todos los procesos de enseñanza-aprendizaje.

A pesar de su posición central, en la mayoría de las disciplinas académicas existe una falta de consenso en cuanto al término, aunque en la mayoría de los casos se incluyen el razonamiento, el cuestionamiento de la información recibida y la consideración de hipótesis (Went \& Ase, 2015, p. 839). En este estudio, hemos elegido referirnos al pensamiento crítico en los términos de Ennis, Lipmman ( $c f$. Solbes, 2013) y Palacios (2017), quienes lo definen como un pensamiento razonable, reflexivo, capaz y responsable que conduce al juicio porque se apoya en los criterios, se autoevalúa y contextualiza. La formación a partir del pensamiento crítico facilita al futuro maestro la creación de estructuras sistematizadas y validadas desde estándares intelectuales, lejos de prejuicios, creencias u opiniones infundadas. En relación con esto, Palacios afirma que un estudiante universitario se convierte en pensador crítico cuando desarrolla con éxito las capacidades de análisis, síntesis, abstracción, generalización y cuestionamiento constante de la realidad y el contexto de enseñanza-aprendizaje (Palacios et al., 2017). 
En el área de la Didáctica de las Ciencias Sociales, Díaz, Santisteban y Cascajero (2013), proponen la formación en pensamiento crítico como estrategia para afrontar los retos de la sociedad de la información, siendo capaz de argumentar con coherencia y emitir juicios fundamentados. Especialmente, la Educación para la Ciudadanía y Derechos Humanos (en adelante, EpC/EDH) exige al docente afrontar en el aula contenidos abstractos, tales como la sensibilización, concienciación, democracia, identidad. La materia requiere el desarrollo de destrezas para argumentar, explicar, analizar y debatir; de forma que acreciente la participación de su alumnado.

Desde la firma, en 2010, de la Carta del Consejo de Europa sobre la Educación para la Ciudadanía Democrática y la Educación en Derechos Humanos, en España, los maestros de EpC/EDH vienen implantando el currículo bajo la observancia de la opinión pública y, en ocasiones, a pesar de la desconfianza generada por la confrontación ideológica y política en las familias (Gómez \& García Ruiz, 2019). Por otro lado, el bajo nivel de participación democrática de la ciudadanía española es un tema ampliamente tratado tanto en la literatura científica como en el último informe sobre Educación para la Ciudadanía en las aulas (Gómez, 2009; Eurydice, 2017). Además, la presencia de EpC/EDH en el Grado de Educación Primaria es testimonial. Estas circunstancias influyen en ética profesional que forja el maestro en formación desde sus primeras experiencias, siendo ésta un indicador de la calidad de la educación de un país (Gatti, 2009).

Un docente que se propone educar bajo la sospecha de un sector de la ciudadanía acaso necesita más que nunca desarrollar una práctica reflexiva que le permita conformar su identidad profesional, pues esta determinará su práctica diaria en el aula. Esa imagen se elabora partir de su faceta personal, constituida por los propios valores, habilidades y conocimientos; y, la dimensión social, conformada por la percepción de los otros, entre los cuales el alumnado desempeña un rol especialmente relevante (Prieto, 2004; Álvarez, 2004; Bolívar, 2007; Ibarra, 2014).

Cuando el pensamiento crítico se lleva a cabo sobre la práctica docente, podemos hablar de práctica reflexiva. Esta implica un reconocimiento del papel activo del docente en formación, capaz de formular los propósitos y los fines de su trabajo, con capacidad para el desarrollo del currículo y reformar la escuela. En este sentido, Cristina del Moral cuando sostiene que la práctica reflexiva se ve favorecida por la formación en competencias (Del Moral, 1997). El estudio de las habilidades profesionales adquiridas por los estudiantes formados a partir de pensamiento crítico ha llevado a Parras a afirmar que tiene incidencia directa en su fluidez verbal (Parras, 2013).

En general, se espera que el futuro docente aprenda a utilizar el pensamiento crítico de forma bidimensional. En primer lugar, como generador de práctica docente reflexiva, ya que fomenta el análisis y la evaluación desde la primera formación y a lo largo de su trayectoria profesional. Por otra parte, como metodología intrínseca al proceso de enseñanza-aprendizaje de EpC/EDH capaz de generar clases dinámicas. Entre otras cosas, el pensamiento crítico facilita al docente la argumentación y le predispone a fundamentarlos desde el currículo y la literatura científica. Asimismo, como instrumento didáctico, le permite la valoración, observación y reflexión sobre el proceso de enseñanza-aprendizaje. Y, además, agiliza la adquisición de 
conceptos y la resolución de problemas de forma colaborativa, convirtiéndolo en protagonista de su propio pensamiento.

Consideramos que la primera formación docente es una etapa crucial en la que, si nuestro alumnado interioriza la práctica reflexiva como forma de trabajo en el aula, los beneficios del aprendizaje permanente estarán garantizados. Por ese motivo, diseñamos un proyecto de innovación educativa en la formación de maestros de EpC/EDH, Maestras y maestros en formación amadrinando y apadrinando Derechos Humanos con los alumnos del CEIP Almudena Grandes de Málaga (septiembre 2019- junio 2020), que utilizaba el ensayo como instrumento para alcanzar competencias generales y profesionales vinculadas con el pensamiento crítico. Completamos esta estrategia con la elaboración de un póster que les permitiera sintetizar los contenidos abstractos de su texto de forma accesible.

Damos continuidad al ensayo como aliado del conocimiento en la acción de Schön (1983). En esta tradición, el ensayo se ve como el medio en el que se fusionan la generación del conocimiento práctico y el proceso reflexivo. Adicionalmente, trabajamos con el póster en la línea planteada por Santos Guerra para los mensajes icónicos, según la cual las imágenes ayudan al docente a transmitir mensajes porque ayudan a la "percepción, simplicidad y economía" ( $c f$. Puñez, 2017, p. 171). Como recursos abiertos a varias interpretaciones, favorecen la libertad de pensamiento y amplían la información.

Un sujeto formado que práctica el pensamiento crítico contrasta la información; pregunta, indaga e investiga; tiene capacidad de abstracción: analiza, elabora e interpreta conceptos; empatiza, es honesto con las opiniones propias y ajenas; contempla el cambio y la diversidad; establece juicios con prudencia; reconsidera opiniones; y, evalúa y se autoevalúa con claridad. Todas estas destrezas permiten al alumnado alcanzar una comprensión profunda sobre un tema especialmente complejo, como los temas impartidos en EpC/EDH, siendo aplicadas durante la investigación y escritura de un ensayo.

El objetivo de este estudio, que parte del proyecto mencionado con anterioridad, era averiguar si el ensayo es una herramienta ágil para alcanzar competencias personales y profesionales del docente en formación. Este propósito se logró analizando los ensayos y pósters realizados por el alumnado tras un pequeño proyecto de investigación sobre un tema de actualidad vinculado a los Derechos Humanos.

De acuerdo con esta circunstancia, nuestra hipótesis planteaba que el uso del ensayo permite mejorar las habilidades reflexivas del maestro en formación. La capacidad de escribir y pensar críticamente habilita al docente para solucionar problemas en la práctica educativa y le facilita una estructura flexible para construir argumentos, desterrar opiniones e integrarlos en un mismo discurso. Ahora bien, nuestra experiencia como docentes de EpC/EDH nos llevó a proponer la elaboración adicional de un póster que les ayudase a salvar la dificultad de exponer en el aula el contenido abstracto del ensayo.

Nuestro análisis cualitativo confirmó los hallazgos de muchas investigaciones previas en torno al ensayo, ninguna de las cuales se realizó en el contexto de esta disciplina impartida a los futuros docentes de primaria. Por otra parte, el póster también aportó información valiosa, 
revelando la capacidad de nuestro alumnado para construir un "relato" que explique los Derechos Humanos a los niños a partir de la recreación visual de sus argumentos.

\section{Investigaciones previas sobre el uso del ensayo en el aula universitaria}

En los últimos años han proliferado las investigaciones educativas en el aula como consecuencia de la expansión de la enseñanza superior y de las nuevas formas de organizar la educación universitaria que demanda al profesorado resultados de aprendizaje específicos (Wendt y Ase, 2015 p. 840). En la educación superior, la escritura de textos académicos se ha vuelto una práctica habitual para reportar esos resultados pues su elaboración exige investigar, seleccionar y discriminar datos, leer, usar vocabulario específico... (Benion et al., 2019; Brown, 2010; Madsen, 2009; Castro y Sánchez, 2013).

Probablemente, el elevado interés de las investigaciones educativas por el ensayo se deba a la amplia extensión de su uso, especialmente en las universidades europeas, porque su escritura demuestra el dominio de un tema en la mayoría de las áreas (Brown, 2010). Y, aunque las líneas de investigación en torno a la producción de textos académicos se hayan diversificado en los últimos años, casi todos los estudios consultados coinciden en señalar la capacidad del género para desarrollar el pensamiento analítico y crítico.

Por una parte, abundan las investigaciones que valoran distintos aspectos y características formales de los textos presentados por el alumnado, tales como intertextualidad, referencias, perspectiva, el uso del vocabulario disciplinar, la elaboración de argumentos o la organización de ideas (Benion et al., 2019; Madsen, 2009; Brown, 2010; Castro y Sánchez, 2013; Errázuriz, 2019). Igualmente, se han realizado estudios sobre la percepción del alumnado con respecto a la tarea de escribir ensayos (Rayas y Méndez, 2017; Hosein y Rao, 2016), así como sobre los problemas a los que se enfrenta en el momento de su escritura (Carlino, 2004; Wendt y Ase, 2015; Errázuriz et al., 2015).

Conviene subrayar que muchos de estos estudios reportan resultados irregulares, aduciendo que el alumnado, en lugar de realizar trabajos argumentativos y analíticos como se les pide, suelen remitir textos descriptivos (MacLellan, 2004; Castro y Sánchez, 2013), dependientes en exceso de lo literal (Errázuriz, 2019), con escaso procesamiento de la información (Adoumieh, 2014) y estableciendo un escaso o nulo diálogo con su lector potencial (Carlino, 2004).

Enfrentados a la disyuntiva de elegir entre la rebaja de sus expectativas académicas o la mejora de las habilidades escritoras de sus estudiantes, muchos docentes han optado por la organización de cursos de iniciación a la investigación y la redacción (Adoimieh, 2014; Callinan, Zee y Wilson, 2017; Hyland, 1990, Dudley-Evans, 2002). Los resultados que arrojan estas experiencias propugnan la necesidad de familiarizar a nuestro alumnado con la producción textual, un requisito que debe abordarse teniendo en cuenta factores como sus experiencias previas, su capacidad de compromiso y las consecuencias no previstas por el tutor (Wendt y Ase, 2015) como, por ejemplo, el condicionamiento que supone para el alumnado elaborar un ensayo con el tutor en mente, pues este sacrificará sus opiniones personales en aras de escribir aquello que cree que se espera de él (Wendt y Ase, 2015; Adoimieh, 2014; MacLellan, 2004; Hosein y Rao, 2016). 
Por otro lado, existen estudios que defienden que la realización de proyectos de investigación y la posterior plasmación de sus hallazgos en un ensayo influyen en el futuro éxito académico y profesional del alumnado universitario (Meneses, Mata y Ravelo, 2007; MacLellan, 2004). Esto se explica porque la autonomía intelectual adquirida con estas prácticas los prepara para afrontar su participación en el mundo exterior. En el caso concreto de los maestros y maestras en formación, son los docentes con una preparación superior los que desarrollan mejores prácticas con niños en su etapa de desarrollo inicial (Errázuriz, 2019, p. 226).

En lo que respecta a nuestra disciplina, la escritura del ensayo como aprendizaje experiencial para la argumentación tiene el potencial de enseñar a los jóvenes a comprometerse como ciudadanos que enfrentan problemas sociales complejos (Benion et al., 2019, p. 3). Es esa relación del contenido con la realidad lo que posibilita una comprensión profunda del tema estudiado y, para establecer esa correspondencia, nada mejor que el uso de los medios de comunicación de masas (Enríquez et al., 2015, p. 204). En opinión de estos autores, los mass media ayudan a desarrollar en el alumnado una actitud crítica y reflexiva hacia los Derechos Humanos porque les permiten apreciar sus matices, fricciones y contradicciones. Descodificar la construcción del discurso establecido en torno a los Derechos Humanos para legitimar moralmente determinados intereses - posición social, jerarquía política, estabilidad...- se revela esencial para alejar a nuestro alumnado del conformismo de la tutela. Es imperativo enseñarles que la supuesta objetividad de los medios de comunicación obedece a intereses editoriales, políticos o empresariales y la mejor forma de hacerlo es contrastar esa información con fuentes heterogéneas. Solo así alcanzarán una nueva compresión de estos principios garantes de la dignidad humana, no como algo atemporal y estático, sino como construcciones culturales en continua evolución sometidas al constante escrutinio de la esfera pública. En definitiva, usar los medios de comunicación para educar en Derechos Humanos nos permite “[...] desalienar a los jóvenes para ayudarlos a comprender, analizar y juzgar la realidad” (Enríquez et al., 2015, p. 225).

En última instancia, el uso de la prensa en el aula como una estrategia didáctica que potencia y fomenta pensamiento crítico, ha sido ampliamente abordado por Sevillano y Bartolomé (1989), Aguaded (1991), Fernández (1997), Pérez (2006), Murphy (2011), Ruiz (2013), Peinado (2013) y Molina, Rodríguez \& Trigueros (2013), en la medida en que reconoce el trabajo de la prensa en el aula como una estrategia didáctica que potencia y fomenta pensamiento crítico. Además, González y Bernabeú (2009) sostienen que ayuda al docente a identificar las causas que generan cambios en el entorno. Es la prensa, más que ningún otro medio de comunicación, la que genera un análisis crítico de la realidad social y despierta la conciencia ciudadana responsable, activa y comprometida.

\section{Materiales y métodos}

La experiencia de aula en la que basamos nuestra investigación se desarrolló durante el primer semestre del curso 2019/2020. De los alumnos matriculados en la asignatura, que ascendían a 362, participaron en el proyecto el 54\%, (195 estudiantes) pertenecientes a los grupos A, C y E. 
Durante 16 sesiones semanales, se trabajaron documentos institucionales con sesiones teóricas y prácticas, en las que se redactó el ensayo objeto de este estudio. Partiendo de una noticia de prensa relacionada con alguno de los Derechos Humanos (1948), el alumnado indagó y redactó el ensayo siguiendo una plantilla. Finalmente, diseñó un póster derivado del ensayo. Estos productos estaban dirigidos al alumnado de Educación Primaria del CEIP Almudena Grandes, formando parte de una exposición celebrada con motivo del Día Internacional del Derecho a la Educación el 24 de enero de 2020. Además, cada equipo realizó una exposición del ensayo, coevaluada por sus pares, conforme al formato PechaKucha.

Un docente formado, con habilidades para el pensamiento crítico, ofrecerá al grupo clase una práctica docente integral, transferirá habilidades procedimentales, conceptuales y actitudinales que le permitirán formular preguntas y problemas con claridad; gestionar información de forma efectiva, reformular conceptos y ofrecer expectativas de éxito para la resolución del problema planteado.

Tabla 1

Transferencia de habilidades en la práctica docente basada en pensamiento crítico

\begin{tabular}{|c|c|}
\hline \multirow{3}{*}{$\begin{array}{l}\text { Habilidad } \\
\text { procedimental }\end{array}$} & $\begin{array}{l}\text { Contrasta información, busca la verdad; está enfocado en preguntar, indagar e } \\
\text { investigar }\end{array}$ \\
\hline & Desarrolla una práctica docente sistemática \\
\hline & Potencia respuestas fundamentadas desde el trabajo en equipo \\
\hline $\begin{array}{l}\text { Habilidad } \\
\text { conceptual }\end{array}$ & Ofrece capacidad de abstracción: analiza, elabora e interpreta conceptos \\
\hline \multirow{3}{*}{$\begin{array}{l}\text { Habilidad } \\
\text { actitudinal }\end{array}$} & $\begin{array}{l}\text { Empatiza, valora con honestidad opiniones propias y ajenas, contempla el cambio } \\
\text { y la diversidad, establece juicios con prudencia y reconsidera opiniones }\end{array}$ \\
\hline & $\begin{array}{l}\text { Evalúa y se autoevalúa con claridad, a lo largo del proceso de enseñanza- } \\
\text { aprendizaje, mediante una práctica docente reflexiva y formativa. }\end{array}$ \\
\hline & Enseña al alumno a sistematizar, gestionar y evaluar el proceso de pensamiento \\
\hline
\end{tabular}

Fuente: Elaboración propia

Para averiguar si el ensayo en la primera formación docente contribuye a la adquisición de habilidades para el pensamiento crítico, llevamos a cabo una investigación cualitativa dada la naturaleza de nuestros sujetos de estudio, la estrategia de recogida de datos (ensayos), y la necesidad de un método flexible que nos permitiera incorporar nuevas líneas y la recogida de datos adicionales (Mills y Gay, 2019).

Los objetivos específicos de la investigación se exponen a continuación:

1. Averiguar si mejoran las habilidades asociadas al pensamiento crítico

2. Averiguar si alcanzan competencias para la vida laboral, mediante la integración de prácticas reflexivas

3. Averiguar si innova en la apropiación del conocimiento, poniendo énfasis en el aprendizaje centrado en el alumnado

4. Averiguar si desarrolla estrategias de información, sensibilización, conciencia y empoderamiento

El material utilizado fueron los ensayos redactados por los 41 subgrupos creados en las distintas aulas, cada uno de ellos con un mínimo de dos integrantes. Como material complementario se analizaron los pósters derivados del ensayo, pues consideramos que podían 
aportar detalles sobre el desarrollo del pensamiento crítico y sobre la experiencia vivida por nuestro alumnado durante el proceso.

En un primer momento, como herramienta analítica se utilizó una rúbrica de elaboración propia, basada en las competencias generales a desarrollar en la asignatura de Educación para la Ciudadanía y Derechos Humanos del Grado de Educación Primaria de la Facultad de Ciencias de la Educación (Universidad de Málaga). De la misma se derivaron aspectos tales como ortografía, organización de ideas, argumentos, estructura, vocabulario, cohesión, revisión crítica bibliográfica, preguntas de investigación, justificación de la noticia, cooperación, etc. Por tanto, la evaluación de los ensayos permitió aplicar los mismos estándares a todos los grupos, desvelando si estos habían alcanzado comprensión del tema, profundidad del análisis crítico, reflexión sobre las cuestiones abordadas y su habilidad para articular ese conocimiento.

Por su parte, los pósters permitieron apreciar si se cumplían los tres primeros objetivos, aportando información adicional sobre el pensamiento visual. En suma, permitieron comprobar en qué medida coadyuvaban a la plasmación de los argumentos expuestos en los ensayos de forma sencilla y lúdica.

El diseño de este estudio ha tenido algunas limitaciones, pues investigadoras y docentes coinciden, no incluyéndose agentes externos en la evaluación. No obstante, creemos que la proximidad existente con los sujetos de estudio permite identificar detalles sutiles que enriquecen la investigación. Por otra parte, la propia naturaleza del ensayo podría representar un problema pues, si bien pueden apreciarse en el mismo el cumplimiento o no de los objetivos planteados, estos logros resultan difíciles de cuantificar. Y es que, como afirma Chavarría, sistematizar los resultados de este tipo de investigaciones es un proceso arduo e incierto que exige cierto grado de sensibilidad ( $c f$. Binda y Balbastre-Benavent, 2013, p. 181).

\section{4. ¿Es el ensayo un instrumento que contribuye al desarrollo del pensamiento crítico en los futuros docentes?}

Antes de analizar los ensayos, hay que mencionar que, para la mayoría del alumnado, esta era la primera vez que se les pedía realizar una investigación en la que abordaran cuestiones metodológicas y teóricas siguiendo las reglas básicas de la redacción académica, estructurado su trabajo en 4 ejes: una introducción con el planteamiento de una hipótesis de trabajo, el desarrollo expositivo-argumentativo; unas conclusiones o reflexiones finales y una relación de las referencias bibliográficas consultadas.

La escritura académica es uno de los mejores métodos pedagógicos que existen pues para ejercerla, el alumno debe "dominar no solo el lenguaje sino varias destrezas a la vez: búsqueda de información, síntesis de la información, redacción del discurso, elaboración, dominio de las estrategias lectoras y oralidad del discurso como finalidad de su aprendizaje" (Segarra-Báez, 2019, p. 253).

Este proceso de elaboración de un ensayo requiere un ejercicio de indagación en torno a un tema que permite un enfoque personal y creativo. Esa libertad propicia un aprendizaje significativo que Olaizola (2011) relaciona con la resolución de problemas, puesto que se coloca al alumno frente a una situación que debe resolver "mediante la indagación, desarrollando el 
proceso el pensamiento crítico, la creatividad, la autonomía y apropiación del trabajo" (Olaizola, 2011, p. 64).

En este caso, con la finalidad de que el alumnado analizase el objeto del ensayo desde varias perspectivas y reflexionase sobre el tema, se les pidió que contrastaran la noticia original con otras fuentes antes de definir su tema. Una vez concretado, procedieron a la búsqueda de información que les pudiera facilitar el análisis y desarrollo de todos los pasos del trabajo. El requisito era documentar y responder a las preguntas que se iban planteando, haciendo uso de fuentes académicas localizadas tanto en repositorios digitales como físicos. Para el desarrollo del ensayo, los alumnos debían disponer de tiempo, por lo que le dedicaron dos meses y medio en horario de grupo reducido, más el tiempo extra que dedicaron fuera del aula.

En general, todos los grupos seleccionaron dos fuentes diferentes del texto periodístico, siendo capaces de detectar la predisposición ideológica de los medios de comunicación masiva. Además, lograron relacionarlos con varios Derechos Humanos, lo cual concuerda con la complementariedad de estos. Este planteamiento abierto incentiva la indagación y la reflexión continúa y aumenta la motivación del alumnado ya que le permite mayor creatividad.

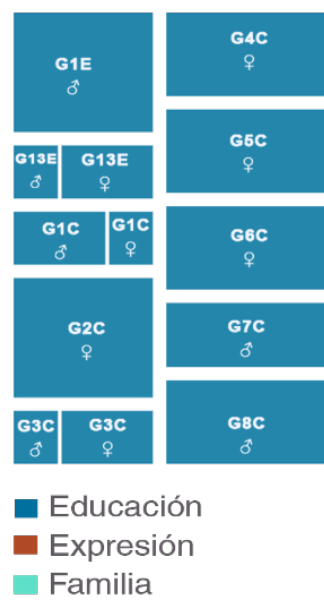

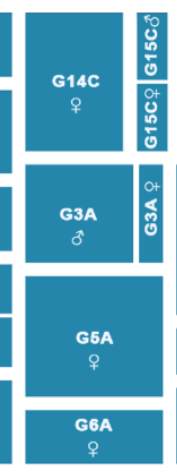

Intimidad

Juego

Movimiento
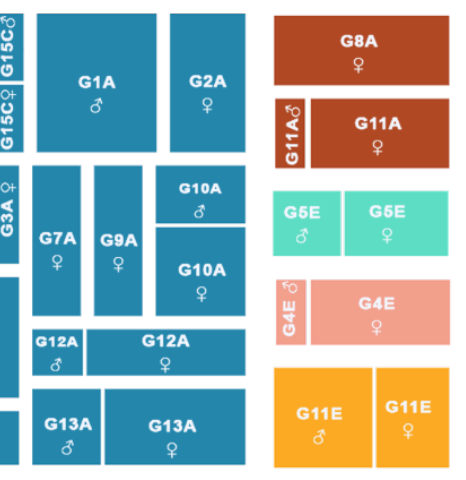

- Libres e iguales

no tortura

- Propiedad

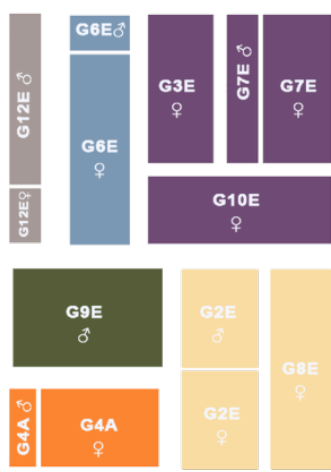

No discriminación Vida

Figura 1. Distribución por equipos según derecho humano trabajado ${ }^{1}$

\section{Fuente: Elaboración propia mediante uso de RawGraphs}

No obstante, todos los grupos tuvieron que elegir solo uno como argumento principal. Las temáticas propuestas en el ensayo final estuvieron relacionadas, mayoritariamente, con el derecho a la educación (27 grupos). Los equipos restantes trabajaron temas diversos como el derecho a la vida (2 grupos), la libertad de expresión (2 grupos), la no discriminación (3 grupos), la no tortura (1 grupo), diferentes formas de familia (1 grupo), disfrute del tiempo libre (1 grupo), derecho a la intimidad (1 grupo), derecho a la propiedad (1), libre circulación (1 grupo) y,

1 En esta gráfica los rectángulos se agrupan por colores, donde el color identifica el derecho humano trabajado reflejado en la leyenda. Por su parte, el tamaño de los rectángulos viene determinado en función de una variable cuantitativa que, en este caso, es la suma del total de miembros integrantes (agrupados por género y grupo). La sigla $G+$ número identifica el nombre del grupo, y la letra final se relaciona con los grupos clase que formaron parte del estudio (A, C o E). 
finalmente, el artículo 1 de la Declaración Universal de los Derechos Humanos, libres e iguales (1 grupo).

El análisis realizado con la ayuda de la rúbrica reveló los siguientes resultados:

En primer lugar, y dado que el ensayo conlleva un proceso introspectivo y crítico, examinamos la capacidad de análisis y reflexión demostrados en el texto. En general, aunque la mayoría plantearon trabajos con una estructura expositiva coherente, no todos indagaron con la misma profundidad en el tema, recurriendo sistemáticamente al uso de un mismo argumento para defender ideas muy distintas. Otro problema detectado estuvo relacionado con el tratamiento personal pues, aunque el ensayo admite "opiniones y juicios de valor sin necesidad de pruebas" (Olaizola, 2011), este no dejaba de ser un texto académico que exigía la construcción de la tesis y argumentos sólidos procedentes de "una investigación, del análisis de lecturas, de la recogida de datos y de una reflexión personal" (Tierno, 2009, p. 132). Hubo trabajos donde los argumentos personales empleados fueron superficiales y, aunque obedecían a su posicionamiento original, desaprovecharon las aportaciones de la investigación documental realizada porque, o bien no la integraron en el corpus ensayístico, o no lo hicieron adecuadamente, aunque hubo excepciones. En casos puntuales, se plantearon ideas que no se desarrollaron a posteriori y, también hubo quien aun consiguiendo relacionar diferentes conceptos en el planteamiento general, no lo resolvió con coherencia, prevaleciendo la sensación de que construyó su ensayo parafraseando párrafos de distinta procedencia.

Con respecto a la competencia básica 2 (CB2), que los maestros en formación "sepan aplicar sus conocimientos a su trabajo y vocación de una formación profesional y posean las competencias que suelen demostrarse por medio de la elaboración y defensa de argumentos y la resolución de problemas dentro de su área de estudio"; y la competencia básica 4 (CB4), que "puedan transmitir información, ideas y soluciones a un público tanto especializado como no especializado" ( Memoria verifica del Grado Educación Primaria, 2013), los resultados fueron diversos. Los errores más comunes obedecieron a incoherencias entre tiempos verbales o género en la construcción de frases que, con frecuencia, eran excesivamente largas y dificultaban la exposición de forma sencilla. Otro aspecto llamativo fue el empleo de lenguaje coloquial en un texto académico. Finalmente, en la estructura y formato se apreciaron errores asociados a la falta de revisión, tales como mezclas de tipografías, colores diferentes de texto, fallos en el formato cita, uso de negritas en párrafos, etc. No obstante, la bibliografía estuvo en general bien elaborada con escasas excepciones. Esto podría explicarse por el taller bibliográfico impartido en el aula en las primeras sesiones prácticas.

También analizamos el póster como herramienta que facilitaría la exposición de argumentos del ensayo de forma sencilla y lúdica, puesto que consideramos que podía aportar información valiosa con respecto a nuestro cuarto objetivo. Al proponer a los alumnos que diseñaran un póster se les motivó a crear una imagen capaz de implementar conceptos relevantes del currículo de EpC/EDH en Andalucía para la sensibilización, concienciación, participación y empoderamiento de un derecho humano. En este punto, éramos conscientes de las habilidades que adquirirían trabajando de forma colaborativa (Castañeda, 2018: 231). Los pósters resultantes, en tanto que imágenes didácticas, se compartieron en una exposición efímera 
celebrada en CEIP Almudena Grandes. De esa manera, cada subgrupo aportó una perspectiva a la exposición general y un buzón de sugerencias creado exprofeso para que el alumnado de quinto de primaria pudiera contribuir con sus impresiones.

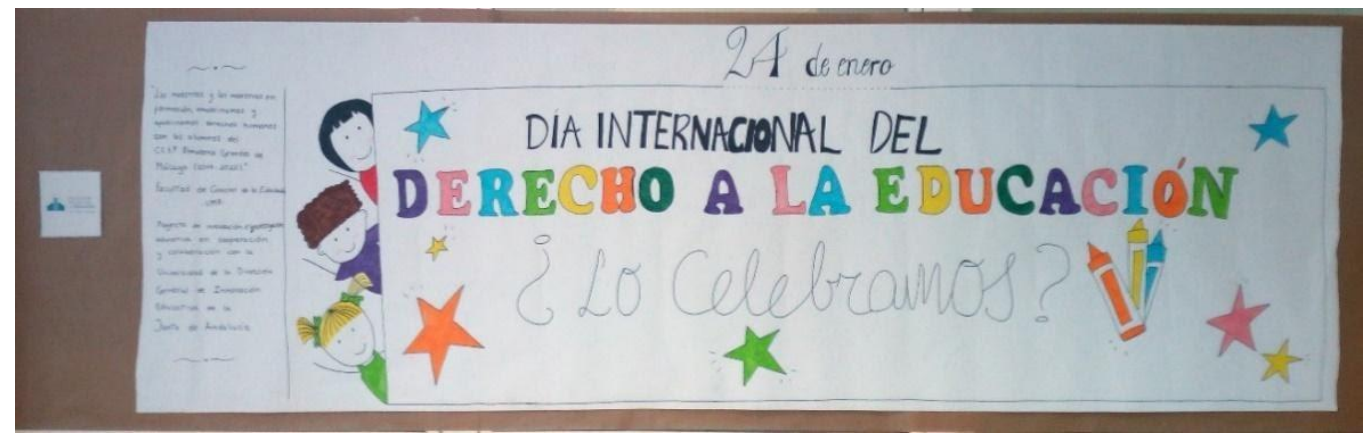

Figura 2. Cartel anunciador de la exposición de pósters realizados por el alumnado de $3^{\circ}$ de Grado de Primaria (UMA) para celebrar el Derecho a la Educación en el CEIP Almudena Grandes (Málaga)

Fuente: Elaboración propia

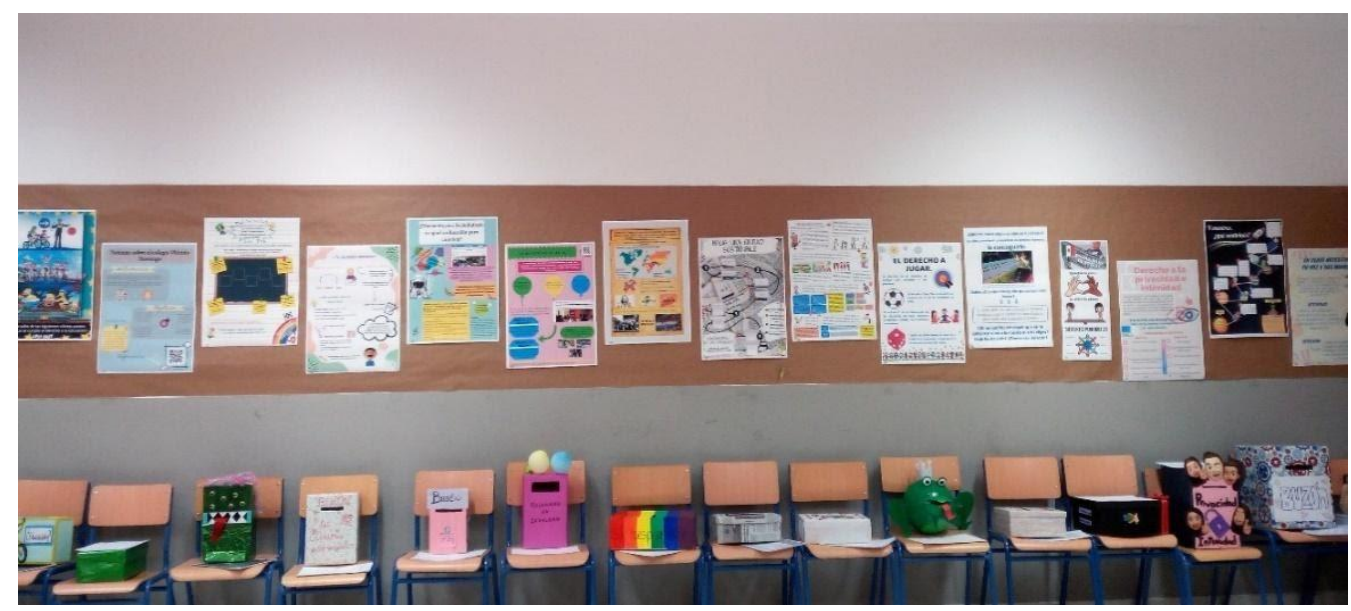

Figura 3. Visión general de la sala de exposiciones del CEIP Almudena Grandes (Málaga).

Fuente: Elaboración propia.

Un análisis preliminar de los pósters, que se ampliará en una segunda etapa del proyecto programada para 2021, reveló que la mayor parte de los equipos de maestros en formación trabajaron la sensibilización (18 grupos), en un intento por despertar en la audiencia cierta empatía ante la causa presentada. La concienciación se usó casi tanto como la primera (17 grupos), consiguiendo que los interlocutores conocieran, comprendieran y valoraran las consecuencias del problema planteado. Este fenómeno coincide con las reflexiones finales de los ensayos, donde suelen plantear las mismas cuestiones que en el póster. Muy alejadas de estas estuvieron las propuestas que apelaban a la acción colectiva para modificar una situación que perciben como injusta o inmoral (4 grupos), coincidentes con temáticas como el acoso escolar, el derecho a la vida, la igualdad o educación. Finalmente, 2 equipos se propusieron informar/documentar exclusivamente; estos casos se explican porque los equipos de trabajo 
tomaron en consideración que su propuesta introducía un concepto que podía resultar especialmente abstracto para los alumnos de 5o del CEIP Almudena Grandes. A modo de ejemplo hay que mencionar que un equipo de maestras en formación se propuso informar/documentar sobre la ratio y su relación con el Derecho a la Educación.

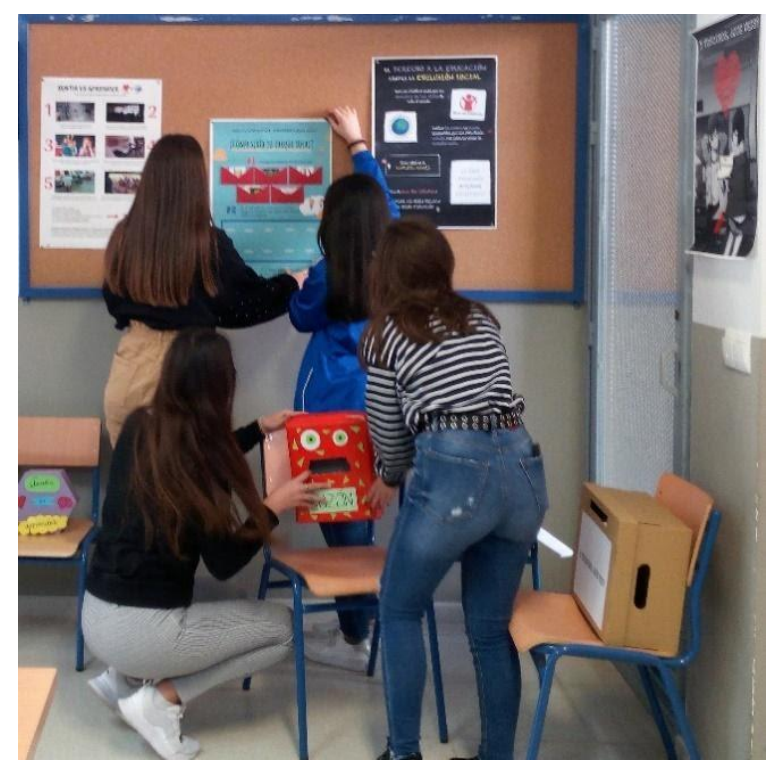

Figura 4. Alumnas de 3o de Grado de Primaria (UMA) durante el montaje de la exposición.

\section{Fuente: Elaboración propia}

\begin{tabular}{|c|c|}
\hline DEBILIDADES & AMENAZAS \\
\hline $\begin{array}{l}\text { - Los libros de texto son básicamente teóricos en la } \\
\text { asignatura, por lo que es imposible que los alumnos } \\
\text { comprueben que conocen sus derechos. Es una forma de } \\
\text { comportarse respecto a los demás. } \\
\text { - El poco tiempo del que dispone la sesión puede suponer } \\
\text { que la actividad no se desarrolle completamente. }\end{array}$ & $\begin{array}{l}\text { - Una de las amenazas principales es la falta de } \\
\text { valoración de la asignatura, seguida de una falta de } \\
\text { apoyo y de recursos en la práctica educativa. } \\
\text { - Como consecuencia del poco tiempo que tenemos } \\
\text { para desarrollar la actividad, es posible que los } \\
\text { alumnos no puedan reflexionar profundamente acerca } \\
\text { de la cuestión del póster. }\end{array}$ \\
\hline FORTALEZAS & OPORTUNIDADES \\
\hline $\begin{array}{l}\text { - El pensamiento visual es una estrategia innovadora que } \\
\text { llama la atención del alumnado. } \\
\text { - Es un tema actual e importante, ya que nos afecta como } \\
\text { ciudadanos/as. } \\
\text { - Esta actividad puede ofrecer a los alumnos y alumnas la } \\
\text { posibilidad de pararse a pensar acerca del concepto de } \\
\text { educación. }\end{array}$ & $\begin{array}{l}\text { - La asignatura tiene el potencial suficiente para llevar } \\
\text { a cabo sesiones prácticas que pueden resultar } \\
\text { interesantes y didácticas de cara a la interpretación y } \\
\text { asimilación de conceptos. } \\
\text { - Trabajar el pensamiento visual ayuda a conseguir un } \\
\text { aprendizaje significativo, facilitando el dinamismo del } \\
\text { proceso de aprendizaje y la adquisición de } \\
\text { conocimientos. } \\
\text { - La posibilidad de trabajar la asignatura de forma } \\
\text { interdisciplinar, involucrando para ello al resto del } \\
\text { profesorado, aumentando el compromiso, la } \\
\text { repercusión e influencia de la Educación para la } \\
\text { Ciudadanía y el conocimiento de los Derechos } \\
\text { Humanos. }\end{array}$ \\
\hline
\end{tabular}

Figura 5. Análisis DAFO elaborado por un equipo de trabajo de maestros en formación
Fuente: Educación para la Ciudadanía y Derechos Humanos Campus Virtual UMA https://ccedu.cv.uma.es/


El mismo póster sirvió como soporte a la puesta en común de su trabajo con el grupo aula. El proceso de evaluación por pares y la retroalimentación de sus compañeros fue una experiencia de aprendizaje muy valiosa, porque les obligó a reflexionar sobre diferentes formas de abordar un tema y les enseñó a comunicar ideas. De ese modo, se generó un aprendizaje colaborativo proporcionando "múltiples modos de apropiación del conocimiento en una persona y a la vez poder atender las diferentes necesidades de aprendizaje propias de la diversidad cultural actual" (Reindhart, 2010, p. 187).

Otro de los aspectos determinantes fueron las conclusiones. Se premió el uso de una matriz DAFO (Figura 5) para facilitar la construcción de argumentos conclusivos con diversas expectativas antes que conclusiones lineales en un único argumento. A la hora de explicar los beneficios de esta metodología, resultó especialmente útil comentar que la propuesta desechaba el golpe de suerte.

\section{Discusión y conclusión}

El balance general del estudio pone de manifiesto que el ensayo como instrumento para la primera formación docente en pensamiento crítico favorece la adquisición de habilidades procedimentales, conceptuales y actitudinales presentes en las competencias generales y específicas en el plan de estudios, memoria verifica, de la materia de Educación para la Ciudadanía y Derechos Humanos en el Grado de Educación Primaria de la Universidad de Málaga. La redacción del ensayo coadyuba para que el aprendizaje del alumnado sea reflexivo y por competencias, pues la escritura es un medio de construcción y comunicación de conocimiento (Meneses, Salvador y Ravelo, 2007). Asimismo, asume la responsabilidad en la propia formación porque, tanto las lecturas previas como los procesos de composición del texto, requieren una planificación y revisión constantes. Finalmente, favorece la alfabetización académica en la disciplina cursada (Legget, el al., 2014; Errázuriz et al., 2015; Castro y Sánchez, 2013).

Respecto al primer objetivo, referente a la mejora de las habilidades asociadas al pensamiento crítico, el uso del ensayo como instrumento para la formación del maestro de EpC/EDH fomentó el pensamiento crítico durante el proceso de elaboración como en concreción en forma de póster. Además, los futuros maestros de educación primaria reconocieron el potencial didáctico de la prensa como fuente y herramienta para motivar el pensamiento crítico. Mediante este trabajo, aprendieron a realizar argumentaciones a partir de un problema real extraído de un periódico (aprendizaje significativo y relevante), integrando en el proceso documentos del acervo didáctico (documentos y recomendaciones legales de UE) y reflexiones sobre los Derechos Humanos. Podría decirse que el principal motivo para hacer uso de la prensa como detonante fue porque de esta manera "se puede demostrar que lo que se enseña en el aula y lo que pasa en el mundo tienen relación" (El mundo, 2016).

En cuanto al segundo objetivo, se implementó la participación activa y responsable de los maestros en formación en cuestiones relativas al ejercicio de la ciudadanía. Primero, los alumnos reflexionaron sobre los derechos estudiados para sí mismos; $y$, segundo, diseñaron un póster para transmitir el conocimiento alcanzado a otros. Además, ejercieron como evaluadores de sus pares durante las exposiciones finales, adoptando un rol profesional. 
En relación con el tercer objetivo, relacionado con la innovación en la apropiación del conocimiento, se apreciaron los siguientes resultados. Por una parte, se inició a los alumnos en la metodología por inducción, puesto que se les propuso que se plantearan una tesis de trabajo para la realización del ensayo que han respetado en el planteamiento de su investigación. Por otra, tanto el tema de trabajo como las competencias didácticas desarrolladas en su elaboración estuvieron vinculadas con los contenidos del currículo.

En lo referente al desarrollo de estrategias visuales, los maestros en formación utilizaron diferentes tácticas para informar, sensibilizar, concienciar o empoderar a los alumnos de $5^{\mathbf{o}}$ Educación para la ciudadanía del CEIP Almudena Grandes. Con la realización de pósters y su posterior exhibición, invitaron a los receptores a participar con sus aportaciones a través de los buzones de sugerencias. Obviamente, realizando esto vincularon también la teoría con la práctica real.

Así pues, esta experiencia permite responder afirmativamente a la pregunta planteada y conocer si se alcanzan los objetivos específicos planteados. En primer lugar, porque es un modo diferente de enseñar Educación para la Ciudadanía en la universidad, basado fundamentalmente en la formación de estructuras de pensamiento crítico integrando el texto periodístico como recurso de aula para favorecer el aprendizaje significativo y relevante. Además, se potencia la creatividad con el ensayo, la creación del póster, las conexiones que se establecen entre Derechos Humanos y temas de actualidad. El alumnado experimenta con un proyecto real que se materializa en la redacción y exposición del ensayo mediante la ficha del profesor, así como a través del diseño y exposición de pósters sobre Derechos Humanos.

Por otra parte, porque permite desarrollar aprendizaje colaborativo y cooperativo, tal y como hemos expuesto en la descripción. La dinámica les muestra el uso de la prensa como herramienta de aula, los inicia en la investigación y les ayuda a desarrollar el pensamiento visual a través del planteamiento del póster. En general puede decirse que han trabajado competencias como la corrección ortográfica y gramatical, la corrección formal, la profundidad del análisis, el rigor en la información, capacidad de síntesis, grado de reflexión crítica, nivel de comprensión de los asuntos abordados, justificación y sentido de la noticia dentro del ensayo, grado de coherencia en la estructura y argumentación, el uso correcto de las fuentes de información y la adecuación a la normativa bibliográfica.

Por último, además de aproximar al alumnado a los contenidos y competencias curriculares del área de Epc/EDH, consigue formar en competencias docentes y didácticas en la medida en que la escritura del ensayo ha servido como instrumento para la formación reflexiva. Por otra parte, propone una colaboración real entre escuela-universidad lo que permite a los alumnos participar activamente en una experiencia real que podría ser replicada en su futuro profesional.

No obstante, aunque hemos podido constatar los beneficios del ensayo como instrumento para la primera formación docente en pensamiento crítico, también hemos detectado deficiencias. Entre los problemas de nuestros alumnos para alcanzar habilidades en comunicación lingüística, destacamos aquellos vinculados con la elaboración y defensa de argumentos y la resolución de problemas dentro de su área de estudio (CB2); así como aquellos relacionados con la transmisión de información, ideas y soluciones a un público tanto especializado como no especializado (CB4). 
Teniendo en cuenta estos resultados consideramos necesario implementar un taller de producción de ensayo para aproximarlos a la escritura académica y potenciar competencias generales como elaboración y defensa de argumentos; resolución de problemas; reunión e interpretación de datos del área de conocimiento de Educación para la ciudadanía y derechos humanos para emitir juicios que incluyan una reflexión sobre temas relevantes de índole social, científica o ética. También pensamos que podría ser útil realizar una entrega previa del ensayo final para que las tutoras lo puedan revisar y hacer propuestas de mejora antes de la entrega definitiva. Además, consideramos que sería una herramienta útil para conocer mejor las dificultades a las que se enfrenta el alumnado, pedirles que entreguen como anexo del ensayo un diario del proceso de elaboración cuya finalidad sería comprender las dificultades que enfrenta el alumnado para generar un texto académico (Errázuriz et al., 2015; Wendt y Ase, 2015). Igualmente, creemos que sería un complemento fundamental el diario del alumnado sobre su trabajo y un diario de las tutoras en el que se reflejen las consultas realizadas. De esa manera, podríamos monitorear mejor el proceso de creación del texto académico y proponer modificaciones cuando sea necesario.

\section{Referencias bibliográficas}

Adoumieh, N. (2014). Escribir desde las disciplinas: una experiencia pedagógica desde las Ciencias Sociales. Paradigma, XXXV (1), 183-213.

Aguaded, J.J. (1991). Profesores dinamizadores de prensa. Huelva: Área de documentación y publicaciones del curso profesores dinamizadores de prensa.

Andalucía. (2015). Área de Educación para la Ciudadanía y los Derechos Humanos (currículo). En Enseñanzas propias de la Comunidad Autónoma de Andalucía para la Educación Primaria (págs. 671-701). Sevilla: Junta de Andalucía.

Basterrechea, B. B. (2017). Informe sobre la ciudadanía de la UE 2017: Fortaleciendo los derechos de los ciudadanos. Bruselas: Unión Europea. Obtenido de https://www.europarl.europa.eu/doceo/document/A-8-2017-0385_ES.html

Bennion, J., Cannon, B., Hill, B., Nelson, R., \& Ricks, M. (2019). Asking the Right Questions: Using Reflective Essays for Experiential Assessment. Journal of Experiential Education, 1-18.

Brown, G. (2010). The Validity of Examination Essays in Higher Education: Issues and Responses. Higher Education Quarterly, 64(10), 276-291.

Callinan, C., van der Zee, E., \& Wilson, G. (2017). Developing essay writing skills: an evaluation of the modelling behaviour method and the influence of student self-efficacy. Journal of Further and Higher Education, 1-15.

Carlino, P. (2004). El proceso de la escritura académica: cuatro dificultades de la enseñanza universitaria. Educere, 8(26), 321-328.

Castro Azuara, M., \& Sánchez Camargo, M. (2013). La expresión de opinión en textos académicos escritos por estudiantes universitarios. RMIE, 18(57), 483-506.

Comisión Europea/EACEA/Eurydice. (2017). La educción para la ciudadanía en el contexto escolar europeo. Luxemburgo: Oficina de publicaciones de la Unión Europea. 
Consejo de Europa (2002). Recomendación (2002)12 del Comité de Ministros a los Estados miembros relativa a la Educación para la Ciudadanía Democrática (adoptada por el Comité de Ministros el 16 de octubre de 2002 en la 812a reunión de los representantes de los Ministros). Consejo de Europa. Obtenido de https://rm.coe.int/09000016804bf0c2

Consejo de Europa. (2002). Manual para la Educación en los Derechos Humanos con jóvenes. Obtenido el 4 de noviembre de 2019, de Consejo de Europa: https://www.coe.int/es/web/compass

Constitución Española. (1978). Boletín Oficial del Estado (311), 29313 - 29424.

Creswell, J. (2014). Research Design. Qualitative, Quantitative and Mixed Methods Approaches Fourth Edition. SAGE Publications.

Domínguez Lázaro, M. (2010). Los medios en el aula: el periodismo como recurso pedagógico. En J. Pérez, Alfabetización mediática y culturas digitales (págs. 931-949). Sevilla: Universidad de Sevilla.

Dudley-Evans, T. (2002). The teaching of the academic essay: Is a Genre Approach posible? En. A. Johns (Ed.). Genre in a classroom. Multiple Perspectives. (pp. 225-236). New Jersey: Lawrence Erlbawm Associates, Publishers

Enríquez Sánchez, J., Pérez Rodríquez, C., Otero León, L., Pérez Domínguez, D., \& Ferrari Nieto, E. (2015). Teoría y práctica educativa de los Derechos Humanos. Valencia: Tirant.

Errázuriz, M. C. (2019). Desempeño escrito de estudiantes de programas de formación inicial docente: ¿Cómo es la calidad del proceso de escritura de sus ensayos? Lengua y habla, 23, 224-242.

Espinel Segura, B. (2011). La prensa en el aula. Cartagena: Ediciones Tecnológica de Bolivar.

Facultad de Ciencias de la Educación Universidad de Málaga. (4 de abril de 2019). Repositorio Facultad de CC.Educación UMA. Obtenido de https://www.uma.es/facultad-de-ciencias-dela-educacion/navegador_de_ficheros/memorias_verifica/listado/

Fernández Martínez, T. (1997). El universo de papel. Trabajamos con el periódico en el aula. Huelva: Grupo Comunicar.

Fernández Martínez, T. (1997). El universo de papel. Trabajamos con el periódico en el aula. Huelva: Grupo comunicar.

Fernández, F. (2002). El análisis de contenido como ayuda metodológica para la investigación. Revista de Ciencias Sociales 2(96), 35-53

Flores, M. (2004) Implicaciones de los paradigmas de investigación en la práctica educativa. Revista Digital Universitaria 5(1), 2-9.

Foro Europeo de la Juventud. (2002). Life-wide learning for active citizenship. Bruselas: European Youth Forum.

Georgesku, D. (2008). Carta para la educación global. En M. Carvalho da Silva (coord.), Pautas para una educación global. Manual para que los educadores comprendan e implementen la educación global (págs. 73-88). Lisboa: Centro Norte-Sur del Consejo Europeo.

Gómez, E. (2007). La Educación para la ciudadanía y los programas de Didáctica de las Ciencias Sociales. En R. Ávila, J. López, \& E. Fernandez, Las competencias profesionales para la enseñanza-aprendizaje de las Ciencias Sociales ante el reto europeo y la globalización (pp 
187-204). Bilbao: Asociación Universitaria de Profesores de Didáctica de las Ciencias Sociales.

Gómez, E., \& García Ruiz, C. (2019). Educación para la ciudadanía en España. Una asignatura para la confrontación ideológica y política. Didáctica de las ciencias experimentales y sociales (37), 51-66.

González Briones, E.; Bernabéu, N. (2009). La noticia y el reportaje. Madrid: Ministerio de Educación-CIDE.

Hernández, A.M.; García, C.R.; De la Montaña, J.L. (Coord.) (2015). Una enseñanza de las ciencias sociales para el futuro. Recursos para trabajar la invisibilidad de personas, lugares y temáticas. Cáceres: Universidad de Extremadura- AUPDCS.

Hosein, A., \& Rao, N. (2016). Students' reflective essays as insights into student centredpedagogies within the undergraduate research methods curriculum. Teaching in Higher Education, 1-17.

Hyland, K. (1990). A Genre Description of the Argumentative Essay. RELC Journal (21), 66-78.

Legget, J., Losekoot, E., Neill, L., \& Wood, Y. (2014). Are Pósters Worth the Paper They Are Printed On? SCHOLE: A Journal of Leisure Studies and Recreation Education, 29 (1), 91-101.

Maclellan, E. (2004). How reflective is the academic essay? Studies in Higher Education, 29(1), 7589.

Madsen, W. (2009). "All at sea": An Activity Theory analysis of first year nursing students learning to write academic essays. Studies in Learning, Evaluation Innovation and Development, 6(1), 38-50.

Maiztegui, C., \& Eizaguirre, M. (2008). Desde la teoría: reflexiones sobre la ciudadanía y la educación ciudadana. En Ciudadanía y educación: de la teoría a la práctica (pp. 13-54). Bilbao: Universidad de Deusto.

Memoria Verificada del Grado de Educación Primaria de la Universidad de Málaga (2013). Obtenido de https://n9.cl/7my37

Meneses Báez, A., Salvador Mata, F., \& Ravelo contreras, E. (2007). Descripción de los procesos cognoscitivos implicados en la escritura de un ensayo. Acta colombiana de psicología, 10(1), 83-98.

Mills, G. E., \& Gay, L. R. (2019). Educational research: Competencies for analysis and applications. Pearson: New Jersey

Molina Puche, S., Rodríguez Pérez, R., \& Trigueros, F. (2013). La prensa como recurso didáctico en Educación Primaria. Reflexiones a partir de su uso por alumnos universitarios. En A. Santisteban, J. Diaz Matarranz, Cascajero, A. (Coord.) Medios de comunicacion y pensamiento crítico: nuevas formas de interacción social (pp. 165-173). Alcalá de Henares: AUPDCS; Universidad de Alcalá de Henares.

Murphy, J. (2011). Más de cien ideas para enseñar historia: primaria y secundaria. Barcelona:Grao. Naciones Unidas (1948). La Declaración Universal de Derechos Humanos. Naciones Unidas.

Núñez Galiano, P.; Flores, P. (2010). Experiencia metodológica en el aprendizaje de competencias desde las didácticas específicas en la formación del profesorado de primaria. En Varios, La 
Docencia en el nuevo Escenario del Espacio Europeo de Educación Superior, (pp. 459-463). Universidad de Vigo.

Olaizola, A. (2011). El ensayo como herramienta en la enseñanza y el aprendizaje de la escritura académica. Reflexión Académica en Diseño y Comunicación, XVI, 61-66. Obtenido de https://fido.palermo.edu/servicios_dyc/publicacionesdc/vista/detalle_articulo.php?id_art iculo $=6480 \&$ \&id_libro $=270$

Parra, D.; Segarra, J.R. (2013). La incidencia del discurso de los medios en las representaciones socioculturales de los futuros maestros: un reto para la didáctica crítica en J.J. Díaz; A. Santisteban; Cascajero, A. (20213). Medios de comunicación y pensamiento crítico. Nuevas formas de interacción social (pp. 77-89). AUPDCS-Universidad de Alcalá de Henares.

Peinado, M. (2013). El papel de los medios de comunicación y la formación del pensamiento crítico en J.J. Díaz; A. Santisteban; Cascajero, A. (20213). Medios de comunicación y pensamiento crítico. Nuevas formas de interacción social (pp. 59-67). AUPDCS -Universidad de Alcalá de Henares.

Puñez Lazo, N. (2017). El Pensamiento visual: una propuesta didáctica para pensar y crear. Horizonte de la Ciencia, 7(12), 161-177.

Ramis, A., \& Peña, M. (2019). Educar para la ciudadanía. San Salvador: INFOD.

Redacción El Mundo. (2016). Razones para usar el periódico en la educación. El Mundo, pág. n.p. Obtenido de https://www.elmundo.es/blogs/elmundo/mejoreducados/2016/04/18/razones-parausar-el-periodico-en-la.html

Ruiz Varela, D. (2013). Ideas para un aprendizaje crítico y creativo en CCSS integrando la prensa y nuevas tecnologías en J. J. Díaz; Santisteban, A.; Cascajero, A. (Coord.). Medios de comunicación y pensamiento crítico. Nuevas formas de interacción social (pp. 67-77). AUPDCS - Universidad de Alcalá de Henares.

Santos Guerra, M. A. (1984) Imagen y Educación. Madrid: Anaya

Segarra-Báez, I. (2019). El ensayo como herramienta y estrategia del aprendizaje. Revista Innova Educación, 1(2), 252-256.

Segovia, J.D. (2003). Educar ciudadanos críticos con los medios de comunicación. Comunicar, 21, 101-108.

Sevillano, M. L.; Bartolomé, D. (1989). Prensa: su didáctica, teoría, experiencias y resultados. Madrid: UNED.

Tierno, B. (2009). Las mejores técnicas de estudio. Madrid: Temas de hoy.

UNESCO (2016). Educación 2030: Declaración de Incheon y Marco de Acción para la realización del Objetivo de Desarrollo Sostenible 4: Garantizar une educación inclusiva y equitativa de calidad y promover oportunidades de aprendizaje permanente para todos. Obtenido de UNESDOC: https://unesdoc.unesco.org/ark:/48223/pf0000245656_spa

Unidos por los Derechos Humanos (2015). Dando vida a los Derechos Humanos [Kit educativo]. Obtenido de https://www.unidosporlosderechoshumanos.es/what-are-humanrights/brief-history/ 
Unión Europea (2005). La Educación para la Ciudadanía en el contexto escolar europeo. Obtenido de EURYDICE: https://www.oei.es/historico/valores2/055ES.pdf.

Unión Europea (2010). Carta del Consejo de Europa sobre la Educación para la Ciudadanía Democrática y la Educación en Derechos Humanos. Obtenido de Consejo de Europa: https://rm.coe.int/1680487829

Villablanca, M. (2015). Diagnóstico de la escritura de un ensayo de alumnos novatos de Pedagogía en el campus Villarica UC, Chile. Perfiles Educativos, XXXVII (150), 76-90.

Wendt, M., \& Åse, C. (2015). Learning dilemmas in undergraduate student independent essays. Studies in Higher Education, 40(5), 838-851. 